\title{
Resonant-state expansion of dispersive open optical systems: Creating gold from sand
}

\author{
E. A. Muljarov and W. Langbein \\ School of Physics and Astronomy, Cardiff University, Cardiff CF24 3AA, United Kingdom \\ (Received 5 October 2015; revised manuscript received 14 January 2016; published 9 February 2016)
}

\begin{abstract}
A resonant-state expansion (RSE) for open optical systems with a general frequency dispersion of the permittivity is presented. The RSE of dispersive systems converts Maxwell's wave equation into a linear matrix eigenvalue problem in the basis of unperturbed resonant states, in this way numerically exactly determining all relevant eigenmodes of the optical system. The dispersive RSE is verified by application to the analytically solvable system of a sphere in vacuum, with a dispersion of the permittivity described by the Drude and Drude-Lorentz models. We calculate the optical modes converting the sphere material from gold to nondispersive sand and back to gold, and evaluate the accuracy using exact solutions.
\end{abstract}

DOI: 10.1103/PhysRevB.93.075417

Any optical system is characterized by its resonances, which are a cornerstone of physics. The concept of resonant states (RSs) is a mathematically rigorous way of treating the resonances. Formally, RSs are the optical eigenmodes of the system, i.e., the eigensolutions of Maxwell's wave equation, which satisfy the outgoing wave boundary conditions. In open optical systems the RS eigenfrequencies $\omega_{n}$ are generally complex, which physically reflects the fact that the energy leaks out of the system. The real part $\operatorname{Re}\left(\omega_{n}\right)$ gives the position of the resonance, while the imaginary $\operatorname{part} \operatorname{Im}\left(\omega_{n}\right)$ gives its half width at half maximum, also determining the quality factor of the resonance as $Q_{n}=\left|\operatorname{Re}\left(\omega_{n}\right) /\left[2 \operatorname{Im}\left(\omega_{n}\right)\right]\right|$.

We have recently developed the resonant-state expansion (RSE), a rigorous method for calculation of RSs, which is treating perturbations of open optical systems of arbitrary strength and shape [1]. Specifically, we note that the RSE (i) uses the natural discretization in the frequency domain provided by RSs, (ii) reduces the solution of Maxwell's wave equation to a linear matrix eigenvalue problem, and (iii) produces all RSs originating from the basis states in a single calculation, avoiding spurious solutions. This enables the RSE to determine numerically exactly all the RSs in a frequency range of interest, with an accuracy limited by the basis truncation only.

Established computational methods in electrodynamics, such as finite difference in time domain (FDTD) and finite element method (FEM), instead, use an artificial discretization in space and time domain and the approximation imposed by perfectly matched layers (PMLs) at the system boundaries. The FEM determines RSs one by one, by solving iteratively a large eigenvalue equation-it is therefore impractical to verify that all RSs within a complex frequency area have been found. In FDTD, RSs can be found by analyzing the calculated time evolution by a sum of RSs. Only RSs that have been excited in the simulation are visible, and the resulting number of RSs is not well defined. In both methods the spatial discretization and PMLs can give rise to spurious solutions. We have explicitly shown in [2] the advantages of the RSE over FDTD and FEM in terms of accuracy and efficiency, exceeding those of the popular computational methods by orders of magnitude.

The RSE was not available until recently since RSs with complex eigenfrequencies have wave functions, which are exponentially growing in space away from system, and the proper general normalization of such RSs was not known. The issues with the normalization have been discussed recently [3], where the presence of PMLs was used to calculate the normalization. At the same time, the correct normalization is contained in our first work on the RSE [1], and we recently generalized it to an arbitrary surface of integration and to dispersive materials, and developed an exact theory of the Purcell effect [4], almost 70 years after its discovery [5].

So far the RSE has been developed for nondispersive systems of different dimensionality and geometry $[1,2,6-8]$. However, almost all realistic systems, even dielectrics such as glass, have a frequency dispersion of the permittivity. We have recently found [9] that the direct substitution of an Ohm's law dispersion into the nondispersive RSE maintains its linearity. The Ohm's law dispersion can be a reasonable approximation for materials with a permittivity dispersion determined by their $\mathrm{dc}$ conductivity or when the dispersion can be approximated by a term linear in the light wavelength over the frequency region of interest. However, metals are better described by the Drude model [10], and a significant improvement is achieved by adding Lorentzian terms [11], which is further refined by using complex weights (residues) of the frequency poles called critical points (CPs) of the permittivity $[12,13]$. This treatment of dispersion is also used in FEM and FDTD, adding significant computational complexity.

In this paper we present a new fundamental method for calculating RSs in open optical systems, generalizing the RSE to treat arbitrary physical materials, by including the frequency dispersion of the permittivity. We verify the method on exactly solvable plasmonic and dielectric nanoparticles, and show that its efficiency and convergence are similar to those of the nondispersive RSE, and thus better than FEM and FDTD.

We start with a basis of RSs with the wave functions $\mathbf{E}_{n}(\mathbf{r})$ and frequencies $\omega_{n}$ being the eigensolutions of Maxwell's wave equation

$$
\nabla \times \nabla \times \mathbf{E}_{n}(\mathbf{r})=\frac{\omega_{n}^{2}}{c^{2}} \hat{\boldsymbol{\varepsilon}}\left(\mathbf{r}, \omega_{n}\right) \mathbf{E}_{n}(\mathbf{r}),
$$

where $\mathbf{r}$ is the spatial position and $\mathbf{E}_{n}(\mathbf{r})$ satisfy the outgoing wave boundary conditions [7]. Generally, the dispersive permittivity tensor $\hat{\varepsilon}(\mathbf{r}, \omega)$ of an unperturbed open optical system described by Eq. (1) can be treated as an analytic function in the complex frequency plane, which contains a countable number of simple poles and therefore, according to 
Mittag-Leffler theorem, is expressed as

$$
\hat{\boldsymbol{\varepsilon}}(\mathbf{r}, \omega)=\hat{\boldsymbol{\varepsilon}}_{\infty}(\mathbf{r})+\sum_{j} \frac{i \hat{\boldsymbol{\sigma}}_{j}(\mathbf{r})}{\omega-\Omega_{j}},
$$

where $\hat{\boldsymbol{\varepsilon}}_{\infty}(\mathbf{r})$ is the high-frequency value of the permittivity and $\Omega_{j}$ are the resonance frequencies (poles) of the permittivity determining the dispersion, with the weight tensors $\hat{\boldsymbol{\sigma}}_{j}(\mathbf{r})$ corresponding to generalized conductivities of the medium at these resonances. The Lorentz reciprocity theorem requires that all tensors in Eq. (2) are symmetric, and the causality principle requires that $\hat{\boldsymbol{\varepsilon}}^{*}(\mathbf{r}, \omega)=\hat{\boldsymbol{\varepsilon}}\left(\mathbf{r},-\omega^{*}\right)$ [14]. Therefore, for a physically relevant dispersion, each pole of the permittivity with a positive real part of $\Omega_{j}$ has a partner at $\Omega_{-j}=-\Omega_{j}^{*}$ with $\hat{\boldsymbol{\sigma}}_{-j}=\hat{\boldsymbol{\sigma}}_{j}^{*}$, while poles with zero real part of $\Omega_{j}$ have real $\hat{\sigma}_{j}$. We assume here a local response of the material, for which the permittivity only depends on a single coordinate. In real materials, the response is nonlocal, typically on the atomic scale, which is relevant for structure sizes in the nanometer range [15].

The Ohm's law dispersion of the permittivity corresponds to replacing the sum in Eq. (2) by a single term with $\Omega_{0}=0$, and $\hat{\boldsymbol{\sigma}}_{0}(\mathbf{r})$ being the dc conductivity tensor. The Drude model of metals consists of two poles, $\Omega_{0}=0$ and $\Omega_{1}=-i \gamma$, with $\hat{\boldsymbol{\sigma}}_{1}(\mathbf{r})=-\hat{\boldsymbol{\sigma}}_{0}(\mathbf{r})$. An example is given in Fig. 1, approximating the measured complex refractive index $n_{r}(\omega)=\sqrt{\epsilon(\omega)}$ of gold [10] using $\hbar \Omega_{1}=-92.8 i \mathrm{meV}, \hbar \sigma_{1}=-744 \mathrm{eV}$, and $\varepsilon_{\infty}=1$ [3]. Here $\epsilon(\omega)$ is the diagonal element of the uniform permittivity tensor in the metal. The Drude-Lorentz model introduces additional poles at $\omega=\Omega_{j}$ with $j= \pm 2, \pm 3, \ldots$ and complex conductivities $\hat{\boldsymbol{\sigma}}_{j}$. We show in Fig. 1 an established model for gold [12], having two pairs of additional resonances with $\hbar \Omega_{1}=-85.6 i \mathrm{meV}, \hbar \sigma_{1}=-882 \mathrm{eV}$,

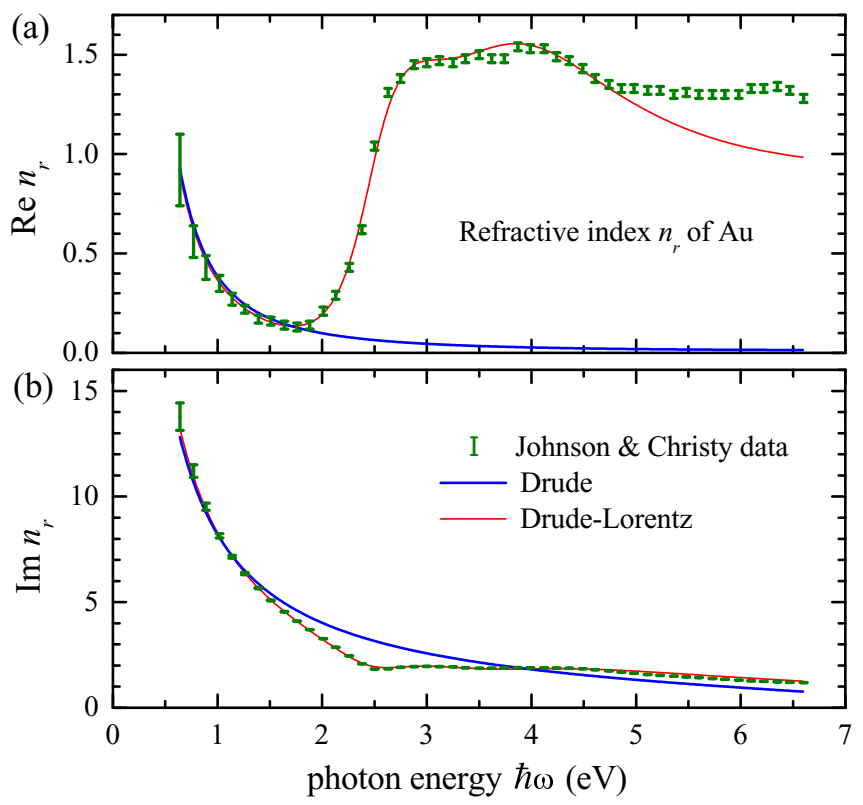

FIG. 1. (a) Real and (b) imaginary parts of the refractive index $n_{r}(\omega)$ of gold, measured by Johnson and Christy [10] (green error bars) and approximated by the Drude model [3] (thick blue lines), and by the Drude-Lorentz model with two pairs of CPs [12] (thin red lines). $\hbar \Omega_{2}=(2.64-0.65 i) \mathrm{eV}, \quad \hbar \sigma_{2}=3.35 e^{i \pi / 4} \mathrm{eV}, \quad \hbar \Omega_{3}=$ $(3.82-1.17 i) \mathrm{eV}, \quad \hbar \sigma_{3}=4.20 e^{i \pi / 4} \mathrm{eV}, \quad$ and $\varepsilon_{\infty}=1.54$. These Drude and Drude-Lorenz models for gold are used in the following for illustration of the dispersive RSE.

The GF of Maxwell's wave equation has the spectral representation

$$
\hat{\mathbf{G}}_{\omega}\left(\mathbf{r}, \mathbf{r}^{\prime}\right)=c^{2} \sum_{n} \frac{\mathbf{E}_{n}(\mathbf{r}) \otimes \mathbf{E}_{n}\left(\mathbf{r}^{\prime}\right)}{2 \omega_{n}\left(\omega-\omega_{n}\right)},
$$

where the sum is taken over all RSs, and $\otimes$ denotes the dyadic product of vectors. Equation (3) requires [4] that the RSs are normalized according to

$$
\begin{aligned}
1+\delta_{0, \omega_{n}}= & \left.\int_{V} \mathbf{E}_{n}(\mathbf{r}) \cdot \frac{\partial\left[\omega^{2} \hat{\boldsymbol{\varepsilon}}(\mathbf{r}, \omega)\right]}{\partial\left(\omega^{2}\right)}\right|_{\omega_{n}} \mathbf{E}_{n}(\mathbf{r}) d \mathbf{r} \\
& +\frac{c^{2}}{2 \omega_{n}^{2}} \oint_{S_{V}}\left(\mathbf{E}_{n} \cdot \frac{\partial \mathbf{F}_{n}}{\partial s}-\mathbf{F}_{n} \cdot \frac{\partial \mathbf{E}_{n}}{\partial s}\right) d S,
\end{aligned}
$$

where $\mathbf{F}_{n}=(\mathbf{r} \cdot \nabla) \mathbf{E}_{n}, V$ is an arbitrary simply connected volume with a boundary surface $S_{V}$ enclosing the inhomogeneity of the system, and the derivative $\partial / \partial s$ is taken along the outer surface normal.

Substitution of the spectral representation Eq. (3) into Maxwell's equation for the GF results in a closure relation and additional sum rules, as shown in Appendix $A$, see Eqs. (A4)-(A6). The sum rules provide additional spectral representations of the $\mathrm{GF}$, one for each pair of poles in the permittivity.

Let us now consider a perturbed system, in which $\hat{\boldsymbol{\varepsilon}}(\mathbf{r}, \omega)$ is replaced by $\hat{\boldsymbol{\varepsilon}}(\mathbf{r}, \omega)+\Delta \hat{\boldsymbol{\varepsilon}}(\mathbf{r}, \omega)$, with the perturbation $\Delta \hat{\boldsymbol{\varepsilon}}(\mathbf{r}, \omega)$ in the form of Eq. (2) described by $\Delta \hat{\boldsymbol{\varepsilon}}_{\infty}(\mathbf{r})$ and $\Delta \hat{\boldsymbol{\sigma}}_{j}(\mathbf{r})$, nonzero only inside the unperturbed system. We find the electric field $\mathbf{E}(\mathbf{r})$ and the eigenfrequency $\omega$ of a perturbed RS using the integral equation

$$
\mathbf{E}(\mathbf{r})=-\frac{\omega^{2}}{c^{2}} \int \hat{\mathbf{G}}_{\omega}\left(\mathbf{r}, \mathbf{r}^{\prime}\right) \Delta \hat{\boldsymbol{\varepsilon}}\left(\mathbf{r}^{\prime}, \omega\right) \mathbf{E}\left(\mathbf{r}^{\prime}\right) d \mathbf{r}^{\prime} .
$$

Expanding the perturbed RS inside the system into the complete basis of unperturbed RSs,

$$
\mathbf{E}(\mathbf{r})=\sum_{n} c_{n} \mathbf{E}_{n}(\mathbf{r}),
$$

and using the different spectral representations of the GF [see Eq. (A9) of Appendix A] for the corresponding terms of the permittivity, the integral equation (5) is converted to the matrix eigenvalue equation

$$
\left(\omega_{n}-\omega\right) \sum_{m}\left[2 \delta_{n m}+V_{n m}(\infty)\right] c_{m}=\omega_{n} \sum_{m} V_{n m}\left(\omega_{n}\right) c_{m},
$$

which is linear in $\omega$, with the perturbation matrix

$$
V_{n m}(\omega)=\int \mathbf{E}_{n}(\mathbf{r}) \cdot \Delta \hat{\boldsymbol{\varepsilon}}(\mathbf{r}, \omega) \mathbf{E}_{m}(\mathbf{r}) d \mathbf{r},
$$

see Appendix B for details. This is the linear dispersive RSE, which treats arbitrarily shaped changes of the permittivity inside the basis system, described by $\Delta \hat{\boldsymbol{\varepsilon}}_{\infty}(\mathbf{r})$ and $\Delta \hat{\boldsymbol{\sigma}}_{j}(\mathbf{r})$. In the absence of dispersion we have $V_{n m}(\omega)=V_{n m}(\infty)$, and Eq. (7) simplifies to $2\left(\omega_{n}-\omega\right) c_{n}=\omega \sum_{m} V_{n m} c_{m}$, which is the matrix eigenvalue equation of the nondispersive RSE [1,2]. 

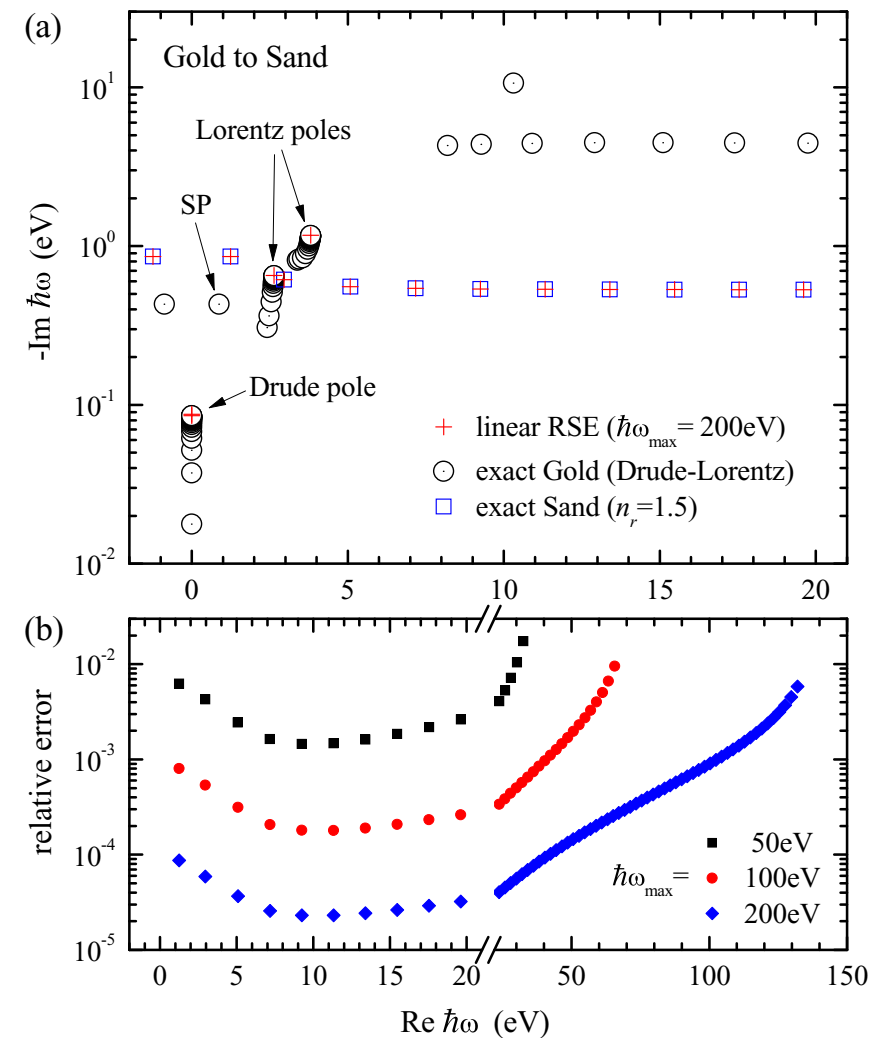

FIG. 2. Results of the linear dispersive RSE converting gold into sand. (a) RS energies $\hbar \omega_{n}$ of the unperturbed system (gold sphere in vacuum using the Drude-Lorentz model, black circles with dots) and the perturbed system (sand sphere in vacuum) for $l=1 \mathrm{TM}$ modes and the sphere radius of $R=200 \mathrm{~nm}$. The perturbed energies are calculated exactly (blue squares) and using the linear RSE Eq. (7) (red crosses) for $\hbar \omega_{\max }=200 \mathrm{eV}$. (b) Relative difference between the RSE and exact eigenenergies, for different values of $\omega_{\max }$ as given.

The linear dispersive RSE is suited for both dispersive and nondispersive unperturbed systems with perturbations, which do or do not add new poles to the permittivity. For every additional nonzero pole of the permittivity, the GF acquires an additional countable infinite number of poles [16], having frequencies asymptotically approaching the permittivity pole. Poles of the permittivity with finite weight in the perturbed system but zero weight in the unperturbed system are included in the basis by taking the limit of the pole weight tending to zero. In this limit, the pole-related RSs have frequencies converging to the pole but refractive indices taking separate discrete values, as detailed below.

To illustrate the linear dispersive RSE and evaluate its convergence, we show in Figs. 2 and 3 the transverse magnetic (TM) eigenmodes of spheres made of a dispersive material (gold) and a nondispersive material (sand, $n_{r}=1.5$ ) in vacuum, and perturbations, which transform gold to sand in Fig. 2 and sand to gold in Fig. 3. We call the nondispersive material sand since it is a fictitious material with a refractive index similar to silica in the visible region. In order to model the fundamental surface plasmon (SP) mode, which is an important mode for applications such as plasmonic biosensing, we choose RSs with the orbital number $l=1$.
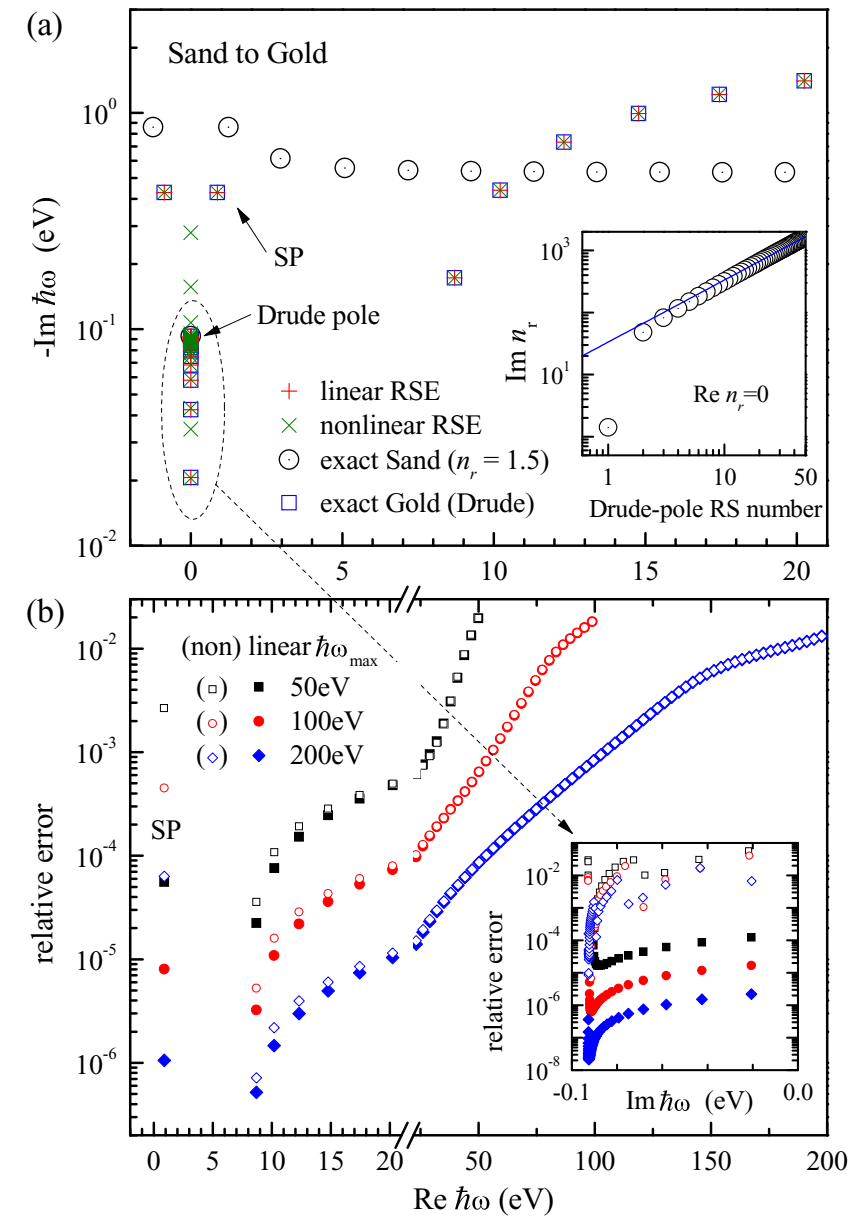

FIG. 3. Results of the dispersive RSE converting sand into gold. (a) RS energies $\hbar \omega_{n}$ of the unperturbed system (sand sphere with $n_{r}=1.5$ in vacuum, black circles with dots) and perturbed system (gold sphere in vacuum using the Drude model) for $l=1 \mathrm{TM}$ modes and $R=200 \mathrm{~nm}$. The perturbed energies are calculated exactly (blue open squares), and using the linear RSE Eq. (7) (red crosses) or the nonlinear RSE Eq. (9) (green crosses). The inset shows the refractive index of degenerate Drude-pole modes in the unperturbed basis sorted in ascending order. The line shows a proportionality between number and index. (b) Relative error of the RSE energies of the perturbed RSs, for both the linear and nonlinear RSE, for different values of $\omega_{\max }$ as given. The inset shows the relative errors of RSs close to the Drude pole, which have purely imaginary energies.

The RSs of the sand and gold spheres in vacuum were taken in the analytic form [2] and normalized according to Eq. (4). Explicit expressions are given in [4]. The radius of the sphere $R=200 \mathrm{~nm}$ is chosen such that both the Drude and the Drude-Lorentz models of the gold permittivity shown in Fig. 1 are approximately valid for the fundamental SP mode at $\hbar \omega \sim(0.88-0.43 i) \mathrm{eV}$ shown in Figs. 2 and 3 by arrows.

We select a finite number $N$ of RSs for the RSE basis, including all RSs satisfying the condition $\left|n_{r}\left(\omega_{n}\right) \omega_{n}\right|<\omega_{\max }$. This excludes RSs having a wave vector in the medium above $\omega_{\max } / c$, which occurs both for large $\omega_{n}$ and for large $\left|n_{r}\left(\omega_{n}\right)\right|$ with $\omega_{n}$ close to the poles of the dispersion. The RSE results for the perturbed eigenmodes are compared with the analytic solutions, and the relative errors are shown 
in Figs. 2(b) and 3(b) for different $\omega_{\max }$, demonstrating a high accuracy given the strong perturbation. Note that for the present geometry, $N$ is approximately proportional to $\omega_{\max }$, with $N=456$ for $\hbar \omega_{\max }=200 \mathrm{eV}$. The observed $1 / N^{3}$ convergence to the exact solution is comparable to that of the nondispersive RSE [1,2], which indicates that the linear dispersive RSE has the same advantages in accuracy and efficiency compared to FDTD and FEM as demonstrated for the nondispersive RSE [2].

Converting gold to sand [Fig. 2(a)] the RSE reproduces the RSs of the nondispersive sand sphere, and additionally produces a number of quasidegenerate RSs at the Drude and Lorentz poles. These RSs are present in the system since in the linear RSE the same poles of the dispersion are considered before and after perturbation. Poles with zero weight lead to a series of RSs, all having the frequencies at the pole position but corresponding to different refractive indices, as exemplified in the inset of Fig. 3(a). For the sphere geometry, they can be calculated analytically by taking the limit of the pole weight to zero in the secular equation. A perturbation that creates a finite weight of the pole lifts the degeneracy of these RSs, as exemplified in Fig. 3(a) converting sand to gold.

We now compare this result with an alternative dispersive RSE approach, which uses a nondispersive system as basis and creates the additional RSs due to the poles of the dispersion via the nonlinearity of the resulting generalized eigenvalue problem. Assuming that the unperturbed $\hat{\boldsymbol{\varepsilon}}(\mathbf{r})$ has no dispersion leaves us with only one sum rule [Eq. (A5) of Appendix A] and one alternative GF representation [Eq. (A9) used for $\Omega_{j}=0$ ]. Using that representation in Eq. (5) results in a nonlinear dispersive $R S E$

$$
2\left(\omega_{n}-\omega\right) c_{n}=\omega \sum_{m} V_{n m}(\omega) c_{m},
$$

which appears as a more direct generalization of the nondispersive RSE [1,2]. For a finite number of poles in the permittivity, Eq. (9) can be written as a polynomial matrix equation in $\omega$. The order $M$ of the polynomial is given by the number of poles in Eq. (2), so for example, $M=1$ for the Ohm's law model (linear matrix problem), $M=2$ for the Drude model (quadratic problem), and $M=6$ for the Drude-Lorentz model with two pairs of CPs. For any finite $M>1$, such a polynomial eigenvalue problem can be solved by linearization [17], extending the size of the matrix problem by a factor of $M$.

We illustrate the nonlinear dispersive RSE using the Drude dispersion of the perturbed system, for which Eq. (9) is a quadratic matrix problem. For the same basis cutoff $\omega_{\max }$ as used for the linear dispersive RSE, the energies of the Fabry-Pérot RSs are reproduced with a similar accuracy, see Fig. 3(b). However, the SP mode has an about two orders of magnitude larger error, and RSs around the Drude pole are also having orders of magnitude larger error as shown in the inset of Fig. 3(b). This can be understood considering that in the nonlinear RSE the basis does not contain the pole RSs, and is therefore less suited to describe RSs close to the permittivity poles. Furthermore, the nonlinear RSE matrix problem is somewhat larger, leading in the present example to approximately twice the computation time. This demonstrates that the linear dispersive RSE is superior to the nonlinear one in determining the electromagnetic properties, specifically close to material resonances, e.g., for plasmons as shown here, but also for phonon polaritons [18] or exciton polaritons [19].

We emphasize that the RSE can treat arbitrary shapes and materials that are located within the basis system. The latter condition is required for the expansion Eq. (6) to be converging, and to retain the outgoing boundary conditions outside of the basis system. As basis system one can use an analytically solvable one, such as a spherical system with homogeneous material as used in this work, but also core-shell systems [20]. However, one can as well use RSs obtained from other solvers. Furthermore, small perturbations of a nonspherical system can be treated using a double-step RSE, which in a first step determines the RSs of the system and their expansions using a spherical basis. A smaller perturbation of the system can then be efficiently calculated by a local perturbation RSE [2] using only few important RSs as basis.

While the present work gives examples for a threedimensionally bounded geometry, the RSE is suited also for planar [8] or wire [7] structures. An extension to other specific geometries such as planar photonic crystals is possible and under development. Beyond electrodynamics, the dispersive RSE approach can be applied to other areas of physics described by linear wave equations, such as quantum mechanics and acoustics, using effective medium models for the underlying materials.

In conclusion, the presented generalization of the RSE to materials with arbitrary frequency dispersion of the permittivity, described by a finite number of simple poles, is extending the applicability of the RSE to general open optical systems, paving the way to its widespread use in electromagnetic simulation.

The data presented in this work are available from the Cardiff University data archive [21].

This work was supported by the Cardiff University EPSRC Impact Acceleration Account EP/K503988/1, and the Sêr Cymru National Research Network in Advanced Engineering and Materials. The authors acknowledge discussions with M. D. Doost and H. Sehmi. E.A.M. acknowledges support from RFBR Grant No. 14-02-00778.

\section{APPENDIX A: DERIVATION OF SUM RULES AND ADDITIONAL SPECTRAL REPRESENTATIONS OF THE GREEN'S FUNCTION OF A SYSTEM WITH DISPERSION}

For an optical system, described by Maxwell's wave equation (1), with a frequency-dependent permittivity $\hat{\boldsymbol{\varepsilon}}(\mathbf{r}, \omega)$, the GF Eq. (3) satisfies the following wave equation with a $\delta$-function source term:

$$
-\nabla \times \nabla \times \hat{\mathbf{G}}_{\omega}\left(\mathbf{r}, \mathbf{r}^{\prime}\right)+\frac{\omega^{2}}{c^{2}} \hat{\boldsymbol{\varepsilon}}(\mathbf{r}, \omega) \hat{\mathbf{G}}_{\omega}\left(\mathbf{r}, \mathbf{r}^{\prime}\right)=\hat{\mathbf{1}} \delta\left(\mathbf{r}-\mathbf{r}^{\prime}\right) .
$$

Substituting Eq. (3) into Eq. (A1) and using Eq. (1), we obtain a closure relation [4]

$$
\sum_{n} \frac{\omega^{2} \hat{\boldsymbol{\varepsilon}}(\mathbf{r} ; \omega)-\omega_{n}^{2} \hat{\boldsymbol{\varepsilon}}\left(\mathbf{r} ; \omega_{n}\right)}{2 \omega_{n}\left(\omega-\omega_{n}\right)} \mathbf{E}_{n}(\mathbf{r}) \otimes \mathbf{E}_{n}\left(\mathbf{r}^{\prime}\right)=\hat{\mathbf{1}} \delta\left(\mathbf{r}-\mathbf{r}^{\prime}\right) .
$$


For the permittivity in the form of Eq. (2), the above equation is transformed, with the help of the algebraic identity

$$
\begin{aligned}
& \frac{1}{\omega_{n}\left(\omega-\omega_{n}\right)}\left(\frac{\omega^{2}}{\omega-\Omega_{j}}-\frac{\omega_{n}^{2}}{\omega_{n}-\Omega_{j}}\right) \\
& =\frac{1}{\omega_{n}-\Omega_{j}}+\frac{\omega}{\omega-\Omega_{j}}\left(\frac{1}{\omega_{n}}-\frac{1}{\omega_{n}-\Omega_{j}}\right),
\end{aligned}
$$

to the expression

$$
\begin{aligned}
\frac{\omega}{2} & \hat{\boldsymbol{\varepsilon}}_{\infty}(\mathbf{r}) \sum_{n} \frac{\mathbf{E}_{n}(\mathbf{r}) \otimes \mathbf{E}_{n}\left(\mathbf{r}^{\prime}\right)}{\omega_{n}} \\
& +\frac{1}{2} \hat{\boldsymbol{\varepsilon}}_{\infty}(\mathbf{r}) \sum_{n} \mathbf{E}_{n}(\mathbf{r}) \otimes \mathbf{E}_{n}\left(\mathbf{r}^{\prime}\right) \\
& +\frac{1}{2} \sum_{n} \sum_{j} \frac{i \hat{\boldsymbol{\sigma}}_{j}(\mathbf{r})}{\omega_{n}-\Omega_{j}} \mathbf{E}_{n}(\mathbf{r}) \otimes \mathbf{E}_{n}\left(\mathbf{r}^{\prime}\right) \\
& +\frac{\omega}{2} \sum_{j} \frac{i \hat{\boldsymbol{\sigma}}_{j}(\mathbf{r})}{\omega-\Omega_{j}} \sum_{n} \frac{\mathbf{E}_{n}(\mathbf{r}) \otimes \mathbf{E}_{n}\left(\mathbf{r}^{\prime}\right)}{\omega_{n}} \\
& -\frac{\omega}{2} \sum_{j} \frac{i \hat{\boldsymbol{\sigma}}_{j}(\mathbf{r})}{\omega-\Omega_{j}} \sum_{n} \frac{\mathbf{E}_{n}(\mathbf{r}) \otimes \mathbf{E}_{n}\left(\mathbf{r}^{\prime}\right)}{\omega_{n}-\Omega_{j}}=\hat{\mathbf{1}} \delta\left(\mathbf{r}-\mathbf{r}^{\prime}\right) .
\end{aligned}
$$

The above closure relation has to be satisfied for any $\omega$. Therefore, it splits into a simpler, frequency-independent closure relation

$$
\frac{1}{2} \sum_{n} \hat{\boldsymbol{\varepsilon}}\left(\mathbf{r}, \omega_{n}\right) \mathbf{E}_{n}(\mathbf{r}) \otimes \mathbf{E}_{n}\left(\mathbf{r}^{\prime}\right)=\hat{\mathbf{1}} \delta\left(\mathbf{r}-\mathbf{r}^{\prime}\right)
$$

and sum rules

$$
\sum_{n} \frac{\mathbf{E}_{n}(\mathbf{r}) \otimes \mathbf{E}_{n}\left(\mathbf{r}^{\prime}\right)}{\omega_{n}}=0
$$

and

$$
\sum_{n} \frac{\mathbf{E}_{n}(\mathbf{r}) \otimes \mathbf{E}_{n}\left(\mathbf{r}^{\prime}\right)}{\omega_{n}-\Omega_{j}}=0 .
$$

The latter holds for every $\Omega_{j}$ contributing to the permittivity given by Eq. (2). Now, using the algebraic identity Eq. (A3) in the form

$$
\frac{1}{\omega_{n}\left(\omega-\omega_{n}\right)}-\frac{1}{\omega \omega_{n}}+\frac{\Omega_{j}}{\omega^{2}\left(\omega_{n}-\Omega_{j}\right)}=\frac{W_{n}^{j}(\omega)}{\omega\left(\omega-\omega_{n}\right)},
$$

where

$$
W_{n}^{j}(\omega)=\frac{\omega_{n}}{\omega} \frac{\omega-\Omega_{j}}{\omega_{n}-\Omega_{j}},
$$

including $W_{n}^{0}(\omega)=1$ for $\Omega_{0}=0$, and combining the GF given by Eq. (3) with the sum rules Eq. (A5) and Eq. (A6), according to the terms in Eq. (A7), we find an additional spectral representation $\hat{\mathbf{G}}_{\omega}^{j}$ of the GF for each pole in the permittivity:

$$
\begin{aligned}
\hat{\mathbf{G}}_{\omega}^{j}\left(\mathbf{r}, \mathbf{r}^{\prime}\right)= & c^{2} \sum_{n} \frac{\mathbf{E}_{n}(\mathbf{r}) \otimes \mathbf{E}_{n}\left(\mathbf{r}^{\prime}\right)}{2 \omega_{n}\left(\omega-\omega_{n}\right)} \\
& -c^{2} \frac{1}{2 \omega} \sum_{n} \frac{\mathbf{E}_{n}(\mathbf{r}) \otimes \mathbf{E}_{n}\left(\mathbf{r}^{\prime}\right)}{\omega_{n}}
\end{aligned}
$$

$$
\begin{aligned}
& +c^{2} \frac{\Omega_{j}}{2 \omega^{2}} \sum_{n} \frac{\mathbf{E}_{n}(\mathbf{r}) \otimes \mathbf{E}_{n}\left(\mathbf{r}^{\prime}\right)}{\omega_{n}-\Omega_{j}} \\
= & c^{2} \sum_{n} W_{n}^{j}(\omega) \frac{\mathbf{E}_{n}(\mathbf{r}) \otimes \mathbf{E}_{n}\left(\mathbf{r}^{\prime}\right)}{2 \omega\left(\omega-\omega_{n}\right)} .
\end{aligned}
$$

Note that the Ohm's law dispersion introduces a $\omega=0$ pole in the permittivity, which leads to the sum rule Eq. (A6) with $\Omega_{0}=0$, identical to Eq. (A5). This sum rule leads the representation $\hat{\mathbf{G}}_{\omega}^{0}\left(\mathbf{r}, \mathbf{r}^{\prime}\right)$ of the GF given by Eq. (A9) with $W_{n}^{0}(\omega)=1$. The $\omega=0$ pole is actually present also in the nondispersive system owing to the longitudinal $\omega_{n}=0$ modes [2]. As a result, the sum rule Eq. (A5) [or Eq. (A6) with $\Omega_{0}=0$ ] holds even without dispersion [7,8], due to the constant term $\hat{\boldsymbol{\varepsilon}}_{\infty}(\mathbf{r})$ in the permittivity. This explains why Ohm's law does not need any significant reformulation of the RSE compared to the nondispersive case and does not require an extension of the basis of RSs [9].

\section{APPENDIX B: DERIVATION OF THE MATRIX EIGENVALUE EQUATION OF THE LINEAR DISPERSIVE RSE}

We consider Maxwell's wave equation

$$
\nabla \times \nabla \times \mathbf{E}(\mathbf{r})=\frac{\omega^{2}}{c^{2}} \hat{\boldsymbol{\varepsilon}}_{p}(\mathbf{r}, \omega) \mathbf{E}(\mathbf{r})
$$

with a perturbed permittivity $\hat{\boldsymbol{\varepsilon}}_{p}(\mathbf{r}, \omega)=\hat{\boldsymbol{\varepsilon}}(\mathbf{r}, \omega)+\Delta \hat{\boldsymbol{\varepsilon}}(\mathbf{r}, \omega)$. The perturbation $\Delta \hat{\boldsymbol{\varepsilon}}(\mathbf{r}, \omega)$ has the form similar to Eq. (2):

$$
\Delta \hat{\boldsymbol{\varepsilon}}(\mathbf{r}, \omega)=\Delta \hat{\boldsymbol{\varepsilon}}_{\infty}(\mathbf{r})+\sum_{j} \frac{i \Delta \hat{\boldsymbol{\sigma}}_{j}(\mathbf{r})}{\omega-\Omega_{j}},
$$

with the poles at the same frequencies $\Omega_{j}$ as in the unperturbed permittivity but with different weights. In Eq. (B1), E(r) is the wave function of a perturbed RS satisfying the outgoing wave boundary condition and $\omega$ is its eigenfrequency. To bring the perturbed problem Eq. (B1) to a linear matrix eigenvalue problem Eq. (7), we use the formal solution for the electric field $\mathbf{E}(\mathbf{r})$ in the form of the integral equation (5), in which we use the unperturbed GF in its different representations Eq. (A9) for the corresponding terms of the permittivity:

$$
\mathbf{E}(\mathbf{r})=-\frac{\omega^{2}}{c^{2}} \int\left[\hat{\mathbf{G}}_{\omega}^{0}\left(\mathbf{r}, \mathbf{r}^{\prime}\right) \Delta \hat{\boldsymbol{\varepsilon}}_{\infty}\left(\mathbf{r}^{\prime}\right)+\sum_{j} \hat{\mathbf{G}}_{\omega}^{j}\left(\mathbf{r}, \mathbf{r}^{\prime}\right) \frac{i \Delta \hat{\boldsymbol{\sigma}}_{j}\left(\mathbf{r}^{\prime}\right)}{\omega-\Omega_{j}}\right]
$$

$$
\times \mathbf{E}\left(\mathbf{r}^{\prime}\right) d \mathbf{r}^{\prime}
$$

Expanding the perturbed RS wave function into the basis of unperturbed ones, Eq. (6), and substituting the spectral representations Eq. (A9) of the GF, we obtain:

$$
\begin{aligned}
\sum_{n} c_{n} \mathbf{E}_{n}(\mathbf{r})= & -\omega^{2} \int\left[\sum_{n} \frac{\mathbf{E}_{n}(\mathbf{r}) \otimes \mathbf{E}_{n}\left(\mathbf{r}^{\prime}\right)}{2 \omega\left(\omega-\omega_{n}\right)} \Delta \hat{\boldsymbol{\varepsilon}}_{\infty}\left(\mathbf{r}^{\prime}\right)\right. \\
& \left.+\sum_{j} \sum_{n} \frac{\omega_{n}}{\omega} \frac{\mathbf{E}_{n}(\mathbf{r}) \otimes \mathbf{E}_{n}\left(\mathbf{r}^{\prime}\right)}{2 \omega\left(\omega-\omega_{n}\right)} \frac{i \Delta \hat{\boldsymbol{\sigma}}_{j}\left(\mathbf{r}^{\prime}\right)}{\omega_{n}-\Omega_{j}}\right] \\
& \times \sum_{m} c_{m} \mathbf{E}_{m}\left(\mathbf{r}^{\prime}\right) d \mathbf{r}^{\prime} .
\end{aligned}
$$


Equating the coefficients at different basis functions $\mathbf{E}_{n}(\mathbf{r})$ results in a matrix equation

$$
c_{n}=-\frac{1}{2\left(\omega-\omega_{n}\right)} \int\left[\omega \mathbf{E}_{n}(\mathbf{r}) \cdot \Delta \hat{\boldsymbol{\varepsilon}}_{\infty}(\mathbf{r})+\omega_{n} \mathbf{E}_{n}(\mathbf{r}) \cdot \sum_{j} \frac{i \Delta \hat{\boldsymbol{\sigma}}_{j}(\mathbf{r})}{\omega_{n}-\Omega_{j}}\right] \sum_{m} c_{m} \mathbf{E}_{m}(\mathbf{r}) d \mathbf{r},
$$

which is equivalent to the linear matrix eigenvalue problem Eq. (7) with the perturbation matrix elements $V_{n m}$ given by Eq. (8).

[1] E. A. Muljarov, W. Langbein, and R. Zimmermann, Europhys. Lett. 92, 50010 (2010).

[2] M. B. Doost, W. Langbein, and E. A. Muljarov, Phys. Rev. A 90, 013834 (2014).

[3] C. Sauvan, J. P. Hugonin, I. S. Maksymov, and P. Lalanne, Phys. Rev. Lett. 110, 237401 (2013).

[4] E. A. Muljarov and W. Langbein, arXiv:1409.6877.

[5] E. M. Purcell, Phys. Rev. 69, 681 (1946).

[6] M. B. Doost, W. Langbein, and E. A. Muljarov, Phys. Rev. A 85, 023835 (2012).

[7] M. B. Doost, W. Langbein, and E. A. Muljarov, Phys. Rev. A 87, 043827 (2013).

[8] L. J. Armitage, M. B. Doost, W. Langbein, and E. A. Muljarov, Phys. Rev. A 89, 053832 (2014).

[9] M. B. Doost, W. Langbein, and E. A. Muljarov, arXiv:1508.03851v1.

[10] P. B. Johnson and R. W. Christy, Phys. Rev. B 6, 4370 (1972).

[11] A. D. Rakić, A. B. Djurišić, J. M. Elazar, and M. L. Majewski, Appl. Opt. 37, 5271 (1998).

[12] P. G. Etchegoin, E. C. Le Ru, and M. Meyer, J. Chem. Phys. 127, 189901 (2007).
[13] A. Vial and T. Laroche, J. Phys. D: Appl. Phys. 40, 7152 (2007).

[14] L. D. Landau, L. P. Pitaevskii, and E. Lifshitz, Electrodynamics of Continuous Media, Vol. 8 of Course of Theoretical Physics, 2nd ed. (Elsevier Butterworth Heinemann, Oxford, 1984).

[15] K. Cho, Optical Response of Nanostructures: Microscopic Nonlocal Theory (Springer, Berlin, 2003).

[16] W. Broer and B. J. Hoenders, J. Phys. A: Math. Theor. 42, 245207 (2009).

[17] J. Higham, D. S. Mackey, N. Mackey, and F. Tisseur, SIAM J. Matrix Anal. Appl. 29, 143 (2006).

[18] S. Dai et al., Science 343, 1125 (2014).

[19] C. E. Platts, M. A. Kaliteevski, S. Brand, R. A. Abram, I. V. Iorsh, and A. V. Kavokin, Phys. Rev. B 79, 245322 (2009).

[20] The Mie Theory: Basics and Applications, Vol. 169 of Springer Series in Optical Sciences, edited by T. W. Wolfram Hergert (Springer-Verlag, Berlin, 2012).

[21] Cardiff University data archive: http://dx.doi.org/10.17035/ d.2016.0008126861. 